\title{
Willingness and Uptake of the COVID-19 Testing and Vaccination in Urban China: An Online Cross- Sectional Survey at Early 2021
}

\section{Suhang Song}

Columbia university https://orcid.org/0000-0003-1934-632X

Shujie Zang

Fudan university

\section{Liubing Gong}

Chizhou Center for Disease Prevention and Control

\section{Cuilin Xu}

Yuhuatai Center for Disease Prevention and Control

Leesa Lin

London School of Hygiene \& Tropical Medicine

\section{Mark R. Francis}

Tampere University

Zhiyuan Hou ( $\boldsymbol{\nabla}$ zyhou@fudan.edu.cn )

Fudan university https://orcid.org/0000-0003-3413-0076

\section{Research Article}

Keywords: Willingness, uptake, COVID-19 testing, COVID-19 vaccination

Posted Date: November 9th, 2021

DOI: https://doi.org/10.21203/rs.3.rs-1048616/v1

License: (c) (i) This work is licensed under a Creative Commons Attribution 4.0 International License. Read Full License 


\section{Abstract}

Background: Regular testing and vaccination are effective measures to mitigate the ongoing COVID-19 pandemic. Few studies have focused on COVID-19 testing and vaccination uptake may change as the pandemic continues. This study aims to examine willingness and uptake of COVID-19 testing and vaccination during a low-risk period of the COVID-19 pandemic in urban China.

Methods: A cross-sectional online survey was conducted among 2244 adults in urban China. Descriptive analyses were performed to compare the respondents' willingness and uptake of COVID-19 testing and vaccination. Multivariate logistic regressions were fitted to investigate the factors associated with willingness and uptake of the two measures.

Findings: In early $2021,52.45 \%$ of the respondents had received or scheduled a COVID-19 test at least once, and a majority (95.63\%) were willing to receive testing. $63.28 \%$ of the respondents had received/scheduled or were willing to receive a COVID-19 vaccine. Willingness and uptake of COVID-19 testing were not associated with socio-demographic characteristics, except for occupation. Being of older age, migrants, having higher educational attainment and secure employment were associated with a higher uptake of COVID-19 vaccination among the surveyed respondents, while willingness to vaccinate was consistent across socio-demographic characteristics among those who had not been vaccinated.

Interpretation: Chinese adults expressed almost universal willingness of COVID-19 testing and relatively low willingness of COVID-19 vaccination at the low-risk period of the COVID-19 pandemic, independent from their socio-demographic characteristics. Maintaining willingness of COVID-19 vaccination are key especially when the pandemic evolved into a low-risk period.

\section{Introduction}

Testing and vaccination are two effective measures to mitigate and prevent the transmission of COVID$19 .^{1-4}$ Testing can be used to diagnose COVID-19 by detecting both symptomatic and asymptomatic patients, and can also trace confirmed cases and their close contacts, especially when outbreaks surge. 3,5 The vaccine is expected to play an important role in preventing serious complications from SARS-CoV-2 infections and establishing herd immunity to protect populations from COVID-19 infections. ${ }^{6}$ COVID-19 vaccinations are being promoted and scaled up globally; in China, the government enacted the emergency use authorization of COVID-19 vaccines in June $2020^{7,8}$, and subsequently approved COVID19 vaccines for general use in December $2020^{8,9}$. By the end of March 2020, the peak of the pandemic has passed in China, and the number of new confirmed cases per day rapidly declined to less than 10; most of these cases were imported from abroad. 4,10,11 With COVID-19 resurging in some regions, mass COVID-19 testing and vaccination strategies have been adopted to track and control sporadic outbreaks in many cities such as Beijing ${ }^{12}$ and Qingdao ${ }^{13}$. Understanding the willingness to receive and uptake of testing and vaccinations can help design and implement policies to improve access to and acceptance of 
COVID-19 tests and vaccination, which is important for effectively promoting pandemic mitigation and prevention strategies.

Previous reviews and empirical studies have focused on estimating the public's willingness to receive and uptake COVID-19 vaccinations. ${ }^{14-26}$ These studies reported that the willingness to vaccinate varied by geographic area $27-34$, socio-demographic characteristics such as age and occupation ${ }^{29-31,35-41}$, and COVID-19 disease and vaccine risk perceptions ${ }^{29,33,42-47}$. A previous survey from China estimated a high willingness to be vaccinated against COVID-19 at the beginning of the pandemic, which declined as the pandemic became normalized due to the reduced perception of COVID-19 risk among the public. ${ }^{48}$ Therefore, it is crucial to continue assessing the public's willingness to be vaccinated as their attitudes and risk perceptions are likely to change over time. Disparities in vaccination uptake also need to be further studied, as only a few studies to date have examined the uptake of the COVID-19 vaccination by population characteristics. COVID-19 testing serves as an important complementary measure to prevent and control spikes in SARS-CoV-2 cases, enabling disease diagnosis and tracing the confirmed cases. ${ }^{49-}$ ${ }^{54}$ Nearly 160 million tests have been performed in China, as of August 6th, $2021 .{ }^{55}$ Since studies on the willingness to be tested and actual uptake of COVID-19 tests are scarce, updated estimates can help identify the population subgroups to be targeted by health education interventions in China.

Therefore, we conducted a cross-sectional survey in early 2021, when COVID-19 testing was being used as a primary measure to detect the sporadic outbreaks of SARS-CoV- 2 cases ${ }^{48}$ and after the COVID-19 vaccine had been officially approved for use among the general public. ${ }^{8}$ This study aims to examine the willingness to receive and uptake of COVID-19 testing and vaccination during the low-risk period of the COVID-19 pandemic in China.

\section{Methods}

\section{Study Design, Population and Sampling}

We conducted an anonymous online cross-sectional survey to collect information on the willingness and uptake of the COVID-19 testing and vaccination and their associated factors among adults aged 18 years and older in two cities (Nanjing and Chizhou) from January 29 to February 4, 2021. Nanjing city in eastern Jiangsu province and Chizhou city in central Anhui province had a gross domestic product (GDP) per capita of CNY 165,681 among 7.10 million population and of CNY 56,217 among 1.62 million population, ${ }^{56}$ respectively, making them good representatives of urban China. ${ }^{57}$ This study employed snowball sampling to enroll the study participants from four to eight community health centers in each city and from the local Centers for Disease Control and Prevention (CDC). Participants could access the questionnaire through a social media platform, WeChat, which has 1.1 billion active users. Those who completed the survey were encouraged to share a link of the questionnaire and invite their colleagues or friends to participate. To avoid repeated participations, each WeChat account was permitted to fill out the questionnaire only once, and only devices having Internet Protocol addresses were able to submit their 
responses successfully. It took about 3-5 minutes to complete the self-administered questionnaire and the respondents were given a gift worth roughly CNY 5 after they completed the survey.

In total, 2250 respondents answered the questionnaire independently and provided e-consent for their participation in the survey. Six incomplete questionnaires or questionnaires completed under two minutes were excluded from the analysis, and a total of 2244 respondents were included in the study. The study was approved by the Institutional Review Board of the School of Public Health, Fudan University (IRB\#2020-12-0861).

\section{Measures}

The self-administered questionnaire was designed based on previous literature and pre-tested among ten respondents, who were excluded from the analysis. The questionnaire included questions on the respondent's socio-demographic characteristics, self-reported health status, awareness of the COVID-19 pandemic, perceived susceptibility and severity of the COVID-19, and willingness to receive and uptake of COVID-19 testing and vaccination.

The outcomes of interest for this study were willingness to receive and uptake of COVID-19 testing and vaccination. Uptake of the COVID-19 test was measured with the question "Have you ever received a COVID-19 test before?" Response options included "already tested," "scheduled," and "haven't been tested or scheduled." Respondents who responded "already tested" or "scheduled" were classified into the uptake group, and were further asked their reasons for receiving testing, including community-wide mass testing led by governments, mandatory testing policies for travel, and personal health needs.

Respondents who responded "haven't been tested or scheduled" were included in the group who haven't uptake the test. The willingness to receive the COVID-19 test was enquired of all participants with a fivepoint Likert scale question, with options including "definitely willing," "probably willing," "not sure," "probably not willing," and "definitely not willing." Responses of "definitely willing" and "probably willing" were classified as "willing", while those who chose the other three options were assigned as "unwilling". Similar to the uptake of COVID-19 testing, uptake of the COVID-19 vaccine was assessed by whether the participants had been vaccinated against COVID-19; those who chose either "vaccinated" or "scheduled" were added to the "vaccinated" group. Only respondents who had not been vaccinated or not scheduled a COVID-19 vaccination were asked for their willingness to be vaccinated in the future. We assigned participants who had not received or scheduled a vaccination to report their willingness to vaccinate as COVID-19 vaccine needs to be received only one time under the policy during the survey time, while COVID-19 tests need to be received multiple times as needed. However, willingness to continue to be tested still matters for those who had already received a COVID-19 test, and thus needed to be analyzed.

The socio-demographic characteristics collected included location, residency, age, gender, marital status, educational attainment, occupation, and annual income. Location, residency and gender were dichotomized into Nanjing city versus Chizhou city, local residents versus migrants, and male versus female, respectively. Participant's age was categorized into four groups, including 18-25, 26-35, 36-45, $\geq 46$ years old. Marital status was classified into single, married and divorced/widow. Educational 
attainment was grouped into middle school or lower, high school or technical secondary school, junior college, and bachelor's degree or higher. Occupation was categorized into four groups: government agency, service industry, manufacturing industry or agriculture, and others. Annual individual income was grouped into $<20,000,20,000-50,000,50,000-100,000,100,000-200,000$ and $>200,000$ Chinese Yuan (CNY). Self-reported health status was assessed on a five-point Likert scale ranging from very good (1) to very poor (5) and dichotomized into "good" (very good and good) versus "poor" (fair, poor and very poor). Awareness of and perceived susceptibility to COVID-19 were also assessed on a five-point Likert scale very high, high, not sure, low, and very low. Respondents who selected the first two options were classified into the "high" group, and those who selected the other three options were assigned to the "low" group. We also asked participants to answer a question on how severe they considered COVID-19 infections to be, "How do you think your symptoms would be if you were infected with COVID-19?", with the following options - severe, moderate, mild, asymptomatic and unsure. These responses were dichotomized into "severe or moderate" versus "mild" (including asymptomatic and unsure).

\section{Statistical Analysis}

Descriptive analyses were performed to compare the characteristics of respondents by their willingness to receive and uptake of COVID-19 testing and vaccination. Since all the assessed factors were categorical variables, chi-square tests were used to compare participant' characteristics across the willingness and uptake groups. Multivariate logistic regression models were fitted to investigate the factors associated with willingness to receive and uptake of COVID-19 testing and vaccination, with the adjusted odds ratio (aOR) and 95\% confidence intervals (Cls) being calculated. The first and the second models regressed the willingness to receive and uptake of COVID-19 testing with the participant's characteristics, respectively; the third and the fourth models regressed the willingness to receive and uptake of COVID-19 vaccination, respectively. Two-sided $\mathrm{P}<0.05$ indicated significance. All analyses were conducted with STATA MP, version 14.0 (StataCorp LLC).

\section{Results}

Table 1 presents a descriptive summary of the characteristics of the survey respondents. Respondents were more likely to be female $(68.81 \%, 1544 / 2244)$, aged $26-35$ years $(46.48 \%, 1043 / 2244)$, local residents $(86.68 \%, 1945 / 2244)$, married $(85.16 \%, 1911 / 2244)$, have a bachelor's degree or higher $(42.02 \%$, $943 / 2244)$, working in a government agency $(34.49 \%, 774 / 2244)$, have an annual income of 50,000 100,000 Chinese Yuan (31.24\%, 701/2244), and report being in good health $(90.24 \%, 2025 / 2244)$. In addition, the majority of respondents portrayed low perceived susceptibility $(93.49 \%, 2098 / 2244)$ and mild perceived severity of COVID-19 $(81.11 \%, 1820 / 2244)$ although they had high awareness of the COVID-19 $(92.78 \%, 2082 / 2244)$. Respondents were almost equally distributed between the two selected cities. 
Table 1

Characteristics of study respondents by the willingness and uptake of COVID-19 test

\begin{tabular}{|c|c|c|c|c|c|}
\hline \multirow[t]{2}{*}{ Characteristics } & \multirow[t]{2}{*}{$\begin{array}{l}\text { Total } \\
(\%)\end{array}$} & \multicolumn{2}{|c|}{ Uptake of COVID-19 test } & \multicolumn{2}{|c|}{$\begin{array}{l}\text { Willingness of } \\
\text { COVID-19 test }\end{array}$} \\
\hline & & $\begin{array}{l}\text { Tested/scheduled } \\
(\%)\end{array}$ & $\begin{array}{l}\text { Not } \\
\text { tested/scheduled } \\
(\%)\end{array}$ & $\begin{array}{l}\text { Willing } \\
(\%)\end{array}$ & $\begin{array}{l}\text { Unwilling } \\
(\%)\end{array}$ \\
\hline Total & $\begin{array}{l}2244 \\
(100)\end{array}$ & $1177(52.45)$ & 1067 (47.55) & $\begin{array}{l}2146 \\
(95.63)\end{array}$ & $98(4.37)$ \\
\hline City & & $P<0.001$ & & $P<0.001$ & \\
\hline Nanjing & $\begin{array}{l}1091 \\
(48.62)\end{array}$ & $626(57.38)$ & $465(42.62)$ & $\begin{array}{l}1024 \\
(93.86)\end{array}$ & $67(6.14)$ \\
\hline Chizhou & $\begin{array}{l}1153 \\
(51.38)\end{array}$ & $551(47.79)$ & $602(52.21)$ & $\begin{array}{l}1122 \\
(97.31)\end{array}$ & $31(2.69)$ \\
\hline Gender & & $P=0.638$ & & $P=0.152$ & \\
\hline Male & $\begin{array}{l}700 \\
(31.19)\end{array}$ & $362(51.71)$ & $338(48.29)$ & $\begin{array}{l}663 \\
(94.71)\end{array}$ & $37(5.29)$ \\
\hline Female & $\begin{array}{l}1544 \\
(68.81)\end{array}$ & $815(52.78)$ & $729(47.22)$ & $\begin{array}{l}1483 \\
(96.05)\end{array}$ & $61(3.95)$ \\
\hline Age (years) & & $P=0.099$ & & $P=0.199$ & \\
\hline $18-25$ & $\begin{array}{l}218 \\
(9.71)\end{array}$ & $119(54.59)$ & $99(45.41)$ & $\begin{array}{l}208 \\
(95.41)\end{array}$ & $10(4.59)$ \\
\hline $26-35$ & $\begin{array}{l}1043 \\
(46.48)\end{array}$ & $519(49.76)$ & $524(50.24)$ & $\begin{array}{l}998 \\
(95.69)\end{array}$ & $45(4.31)$ \\
\hline $36-45$ & $\begin{array}{l}567 \\
(25.27)\end{array}$ & $317(55.91)$ & $250(44.09)$ & $\begin{array}{l}549 \\
(96.83)\end{array}$ & $18(3.17)$ \\
\hline$>=46$ & $\begin{array}{l}416 \\
(18.54)\end{array}$ & $222(53.37)$ & $194(46.63)$ & $\begin{array}{l}391 \\
(93.99)\end{array}$ & $25(6.01)$ \\
\hline Residency & & $P=0.309$ & & $P=0.003$ & \\
\hline Local residents & $\begin{array}{l}1945 \\
(86.68)\end{array}$ & $1012(52.03)$ & $933(47.97)$ & $\begin{array}{l}1870 \\
(96.14)\end{array}$ & $75(3.86)$ \\
\hline Migrants & $\begin{array}{l}299 \\
(13.32)\end{array}$ & $165(55.18)$ & $134(44.82)$ & $\begin{array}{l}276 \\
(92.31)\end{array}$ & $23(7.69)$ \\
\hline Marital status & & $P=0.044$ & & $P=0.352$ & \\
\hline Single & $\begin{array}{l}283 \\
(12.61)\end{array}$ & $168(59.36)$ & $115(40.64)$ & $\begin{array}{l}266 \\
(93.99)\end{array}$ & $17(6.01)$ \\
\hline Married & $\begin{array}{l}1911 \\
(85.16)\end{array}$ & $984(51.49)$ & $927(48.51)$ & $\begin{array}{l}1832 \\
(95.87)\end{array}$ & $79(4.13)$ \\
\hline
\end{tabular}




\begin{tabular}{|c|c|c|c|c|c|}
\hline \multirow[t]{2}{*}{ Characteristics } & \multirow[t]{2}{*}{$\begin{array}{l}\text { Total } \\
\text { (\%) }\end{array}$} & \multicolumn{2}{|c|}{ Uptake of COVID-19 test } & \multicolumn{2}{|c|}{$\begin{array}{l}\text { Willingness of } \\
\text { COVID-19 test }\end{array}$} \\
\hline & & $\begin{array}{l}\text { Tested/scheduled } \\
\text { (\%) }\end{array}$ & $\begin{array}{l}\text { Not } \\
\text { tested/scheduled } \\
(\%)\end{array}$ & $\begin{array}{l}\text { Willing } \\
(\%)\end{array}$ & $\begin{array}{l}\text { Unwilling } \\
\text { (\%) }\end{array}$ \\
\hline Divorced/widow & $\begin{array}{l}50 \\
(2.23)\end{array}$ & $25(50)$ & $25(50)$ & $48(96)$ & $2(4)$ \\
\hline Educational attainment & & $P<0.001$ & & $P=0.343$ & \\
\hline Middle school or lower & $\begin{array}{l}341 \\
(15.20)\end{array}$ & $131(38.42)$ & $210(61.58)$ & $\begin{array}{l}329 \\
(96.48)\end{array}$ & $12(3.52)$ \\
\hline $\begin{array}{l}\text { High school or } \\
\text { technical secondary } \\
\text { school }\end{array}$ & $\begin{array}{l}402 \\
(17.91)\end{array}$ & $163(40.55)$ & $239(59.45)$ & $\begin{array}{l}378 \\
(94.03)\end{array}$ & $24(5.97)$ \\
\hline Junior college & $\begin{array}{l}558 \\
(24.87)\end{array}$ & $289(51.79)$ & $269(48.21)$ & $\begin{array}{l}536 \\
(96.06)\end{array}$ & $22(3.94)$ \\
\hline $\begin{array}{l}\text { Bachelor's degree or } \\
\text { higher }\end{array}$ & $\begin{array}{l}943 \\
(42.02)\end{array}$ & $594(62.99)$ & $349(37.01)$ & $\begin{array}{l}903 \\
(95.76)\end{array}$ & $40(4.24)$ \\
\hline Occupation & & $P<0.001$ & & $P=0.001$ & \\
\hline Government agency & $\begin{array}{l}774 \\
(34.49)\end{array}$ & $582(75.19)$ & $192(24.81)$ & $\begin{array}{l}758 \\
(97.93)\end{array}$ & $16(2.07)$ \\
\hline Service industry & $\begin{array}{l}580 \\
(25.85)\end{array}$ & $273(47.07)$ & $307(52.93)$ & $\begin{array}{l}547 \\
(94.31)\end{array}$ & $33(5.69)$ \\
\hline $\begin{array}{l}\text { Manufacturing industry } \\
\text { or agriculture }\end{array}$ & $\begin{array}{l}302 \\
(13.46)\end{array}$ & $102(33.77)$ & $200(66.23)$ & $\begin{array}{l}290 \\
(96.03)\end{array}$ & $12(3.97)$ \\
\hline Others & $\begin{array}{l}588 \\
(26.20)\end{array}$ & $220(37.41)$ & $368(62.59)$ & $\begin{array}{l}551 \\
(93.71)\end{array}$ & $37(6.29)$ \\
\hline $\begin{array}{l}\text { Annual individual } \\
\text { income }\end{array}$ & & $P=0.001$ & & $P=0.821$ & \\
\hline$<20 k$ & $\begin{array}{l}244 \\
(10.87)\end{array}$ & $107(43.85)$ & $137(56.15)$ & $\begin{array}{l}230 \\
(94.26)\end{array}$ & $14(5.74)$ \\
\hline $20-50 k$ & $\begin{array}{l}373 \\
(16.62)\end{array}$ & $181(48.53)$ & $192(51.47)$ & $\begin{array}{l}359 \\
(96.25)\end{array}$ & $14(3.75)$ \\
\hline $50-100 k$ & $\begin{array}{l}701 \\
(31.24)\end{array}$ & $359(51.21)$ & $342(48.79)$ & $\begin{array}{l}672 \\
(95.86)\end{array}$ & $29(4.14)$ \\
\hline $100-200 k$ & $\begin{array}{l}606 \\
(27.01)\end{array}$ & $339(55.94)$ & $267(44.06)$ & $\begin{array}{l}579 \\
(95.54)\end{array}$ & $27(4.46)$ \\
\hline$>200 k$ & $\begin{array}{l}320 \\
(14.26)\end{array}$ & $191(59.69)$ & $129(40.31)$ & $\begin{array}{l}306 \\
(95.63)\end{array}$ & $14(4.38)$ \\
\hline
\end{tabular}




\begin{tabular}{|c|c|c|c|c|c|}
\hline \multirow[t]{2}{*}{ Characteristics } & \multirow[t]{2}{*}{$\begin{array}{l}\text { Total } \\
\text { (\%) }\end{array}$} & \multicolumn{2}{|c|}{ Uptake of COVID-19 test } & \multicolumn{2}{|c|}{$\begin{array}{l}\text { Willingness of } \\
\text { COVID-19 test }\end{array}$} \\
\hline & & $\begin{array}{l}\text { Tested/scheduled } \\
(\%)\end{array}$ & $\begin{array}{l}\text { Not } \\
\text { tested/scheduled } \\
\text { (\%) }\end{array}$ & $\begin{array}{l}\text { Willing } \\
(\%)\end{array}$ & $\begin{array}{l}\text { Unwilling } \\
\text { (\%) }\end{array}$ \\
\hline $\begin{array}{l}\text { Self-reported health } \\
\text { status }\end{array}$ & & $P=0.382$ & & $P=0.232$ & \\
\hline Good & $\begin{array}{l}2025 \\
(90.24)\end{array}$ & $1056(52.15)$ & $969(47.85)$ & $\begin{array}{l}1940 \\
(95.80)\end{array}$ & $85(4.20)$ \\
\hline Poor & $\begin{array}{l}219 \\
(9.76)\end{array}$ & $121(55.25)$ & $98(44.75)$ & $\begin{array}{l}206 \\
(94.06)\end{array}$ & $13(5.94)$ \\
\hline Awareness of COVID-19 & & $P=0.014$ & & $P<0.001$ & \\
\hline High & $\begin{array}{l}2082 \\
(92.78)\end{array}$ & 1107 (53.17) & $975(46.83)$ & $\begin{array}{l}2007 \\
(96.40)\end{array}$ & $75(3.60)$ \\
\hline Low & $\begin{array}{l}162 \\
(7.22)\end{array}$ & $70(43.21)$ & $92(56.79)$ & $\begin{array}{l}139 \\
(85.80)\end{array}$ & $\begin{array}{l}23 \\
(14.20)\end{array}$ \\
\hline $\begin{array}{l}\text { Perceived susceptibility } \\
\text { of COVID-19 }\end{array}$ & & $P<0.001$ & & $P=0.157$ & \\
\hline High & $\begin{array}{l}146 \\
(6.51)\end{array}$ & $116(79.45)$ & $30(20.55)$ & $\begin{array}{l}143 \\
(97.95)\end{array}$ & $3(2.05)$ \\
\hline Low & $\begin{array}{l}2098 \\
(93.49)\end{array}$ & $1061(50.57)$ & $1037(49.43)$ & $\begin{array}{l}2003 \\
(95.47)\end{array}$ & $95(4.53)$ \\
\hline $\begin{array}{l}\text { Perceived severity of } \\
\text { COVID-19 }\end{array}$ & & $P=0.034$ & & $P=0.696$ & \\
\hline Severe or moderate & $\begin{array}{l}424 \\
(18.89)\end{array}$ & $242(57.08)$ & $182(42.92)$ & $\begin{array}{l}404 \\
(95.28)\end{array}$ & $20(4.72)$ \\
\hline Mild & $\begin{array}{l}1820 \\
(81.11)\end{array}$ & $935(51.37)$ & $885(48.63)$ & $\begin{array}{l}1742 \\
(95.71)\end{array}$ & $78(4.29)$ \\
\hline
\end{tabular}

Figure 1 presents the survey respondent's willingness to receive and uptake COVID-19 testing and vaccination. At the time of the survey, $52.45 \%(1177 / 2244)$ and $23.62 \%(530 / 2244)$ of respondents had received or scheduled at least one COVID-19 test and COVID-19 vaccine, respectively (Figure 1A). Among the respondents who had ever received or scheduled a COVID-19 test, 57.50\% (675/1174) did so because of community-wide mass testing led by governments, followed by mandatory testing policies for travel $(31.35 \%, 368 / 1174)$, and personal health needs $(11.16 \%, 131 / 1174)$ (Figure 2$)$. Concerning willingness to receive COVID-19 tests or vaccines, $95.63 \%$ (2146/2244) reported being willing to receive a COVID-19 test, and $63.28 \%$ (1418/2241) either received, scheduled, or reported being willing to receive a COVID-19 vaccination (Figure 1B). 
Table 1 also contains a descriptive summary of the respondents' characteristics stratified by their willingness to receive and uptake COVID-19 testing. More respondents in Nanjing city had received or scheduled a COVID-19 test at least once $(57.38 \%, 626 / 1091)$, compared to respondents in Chizhou city $(47.79 \%, 551 / 1153)$. About three-fourths $(75.19 \%, 582 / 774)$ of respondents who worked in a government agency had ever received or scheduled a COVID-19 test, than respondents working in service, manufacturing, agriculture, and other industries. Respondents who had completed junior college or received a bachelor's degree or higher had a greater uptake rate of COVID-19 testing-51.79\% (289/558) and $62.99 \%$ (594/943), respectively. However, fewer respondents (39.57\% [294/743]) with lower educational attainment (high school and lower) had ever received/scheduled a COVID-19 test. More than half of the respondents with an annual individual income over 50,000 Chinese Yuan had received or scheduled a test at least once. A similar proportion (53.17\% [1107/2082] of respondents who reported a high level of COVID-19 awareness had ever received or scheduled a COVID-19 test. Across each group, more than $85.80 \%$ of respondents were willing to receive a test.

Table 2 presents the factors associated with willingness to receive and uptake of COVID-19 testing using multivariate logistic regressions. Location, occupation, awareness of and perceived susceptibility to COVID-19 were significantly associated with receiving testing. Compared to respondents living in Nanjing city, fewer respondents in Chizhou city had ever received or scheduled a COVID-19 test (aOR=0.765, 95\% $\mathrm{Cl}=0.619-0.946)$. However, more respondents in Chizhou city were willing to receive the test than in Nanjing city (aOR=2.097, 95\% Cl=1.248-3.524). Compared to respondents who reported working in a government agency, respondents with less secure occupations (service, manufacturing, agricultural or other industries) had a significantly lower uptake and willingness to receive of COVID-19 tests.

Associations between the other socio-demographic factors (i.e., marital status, educational attainment, annual individual income) and the willingness to receive and uptake of COVID-19 testing failed to reach statistical significance in the multivariate analysis. The uptake rate among respondents with a high perceived susceptibility to COVID-19 was nearly three times (aOR: $2.719,95 \% \mathrm{Cl}=1.739-4.251$ ) higher than those with lower perceived susceptibility. The willingness to receive testing among respondents with high COVID-19 awareness was 4.318 times $(95 \% \mathrm{Cl}=2.550-7.314)$ higher than those with low awareness. 
Table 2

Factors associated with the willingness and uptake of COVID-19 test

Uptake of COVID-

19 test (aOR)
$95 \% \mathrm{Cl}$ Willingness of COVID19 test (aOR)

$95 \% \mathrm{Cl}$

City (ref: Nanjing)

Chizhou

$0.765^{\star}$

$\left(0.619-\quad 2.097^{\star \star}\right.$

$0.946)$

$(1.248-$

$3.524)$

Gender (ref: male)

Female

1.116

(0.912 - $\quad 1.436$

1.367)

(0.913 -

2.259)

Age (ref: $18-25$ years)

$\begin{array}{lllll}26-35 & 0.703 & (0.487- & 0.707 & (0.305- \\ & & 1.013) & & 1.641) \\ 36-45 & 0.725 & \begin{array}{l}(0.478- \\ 1.098)\end{array} & 0.750 & (0.272- \\ & & & (0.066) \\ >=46 & 0.832 & (0.537- & 0.429 & 1.189)\end{array}$

Residency (ref: local residents)

Migrants

1.179

(0.893 - $\quad 0.606$

$(0.348-$

1.555)

1.055)

Marital status (ref: single)

Married

0.912

(0.652 - $\quad 1.229$

(0.609 -

1.275)

2.479)

Divorced/widow

0.783

(0.396 - $\quad 1.511$

1.547)

$(0.305$ -

7.494)

Educational attainment (ref: middle school or lower)

\begin{tabular}{|c|c|c|c|c|}
\hline $\begin{array}{l}\text { High school or technical } \\
\text { secondary school }\end{array}$ & 0.862 & $\begin{array}{l}(0.630- \\
1.179)\end{array}$ & 0.552 & $\begin{array}{l}(0.263- \\
1.161)\end{array}$ \\
\hline Junior college & 1.022 & $\begin{array}{l}(0.747- \\
1.398)\end{array}$ & 0.717 & $\begin{array}{l}(0.326- \\
1.576)\end{array}$ \\
\hline Bachelor's degree or higher & 1.252 & $\begin{array}{l}(0.893- \\
1.756)\end{array}$ & 0.686 & $\begin{array}{l}(0.302- \\
1.558)\end{array}$ \\
\hline
\end{tabular}

Occupation (ref: government agency)

${ }^{\star} \mathrm{p}<0.05 .{ }^{* \star} \mathrm{p}<0.01$.

Abbreviation: $\mathrm{aOR}=$ adjusted odds ratio. 


\begin{tabular}{|c|c|c|c|c|}
\hline & $\begin{array}{l}\text { Uptake of COVID- } \\
19 \text { test (aOR) }\end{array}$ & $95 \% \mathrm{Cl}$ & $\begin{array}{l}\text { Willingness of COVID- } \\
19 \text { test (aOR) }\end{array}$ & $95 \% \mathrm{Cl}$ \\
\hline Service industry & $0.307 \star \star$ & $\begin{array}{l}(0.239- \\
0.395)\end{array}$ & $0.403^{\star \star}$ & $\begin{array}{l}(0.212- \\
0.767)\end{array}$ \\
\hline $\begin{array}{l}\text { Manufacturing industry or } \\
\text { agriculture }\end{array}$ & $0.190 * \star$ & $\begin{array}{l}(0.138- \\
0.261)\end{array}$ & 0.570 & $\begin{array}{l}(0.253- \\
1.284)\end{array}$ \\
\hline Others & $0.216^{\star \star}$ & $\begin{array}{l}(0.164- \\
0.283)\end{array}$ & $0.316^{\star \star}$ & $\begin{array}{l}(0.162- \\
0.617)\end{array}$ \\
\hline \multicolumn{5}{|c|}{ Annual individual income (ref: <20k) } \\
\hline $20-50 k$ & 1.207 & $\begin{array}{l}(0.854- \\
1.706)\end{array}$ & 1.295 & $\begin{array}{l}(0.591- \\
2.837)\end{array}$ \\
\hline $50-100 k$ & 1.021 & $\begin{array}{l}(0.739- \\
1.410)\end{array}$ & 1.294 & $\begin{array}{l}(0.640- \\
2.617)\end{array}$ \\
\hline $100-200 k$ & 0.895 & $\begin{array}{l}(0.632- \\
1.267)\end{array}$ & 1.230 & $\begin{array}{l}(0.580- \\
2.609)\end{array}$ \\
\hline$>200 k$ & 0.804 & $\begin{array}{l}(0.539- \\
1.199)\end{array}$ & 1.196 & $\begin{array}{l}(0.500- \\
2.860)\end{array}$ \\
\hline \multicolumn{5}{|c|}{ Self-reported health status (ref: good) } \\
\hline Poor & 1.087 & $\begin{array}{l}(0.795- \\
1.486)\end{array}$ & 0.833 & $\begin{array}{l}(0.436- \\
1.594)\end{array}$ \\
\hline \multicolumn{5}{|c|}{ Awareness of COVID-19 (ref: low) } \\
\hline High & 1.286 & $\begin{array}{l}(0.910- \\
1.817)\end{array}$ & $4.318^{\star *}$ & $\begin{array}{l}(2.550- \\
7.314)\end{array}$ \\
\hline \multicolumn{5}{|c|}{ Perceived susceptibility of COVID-19 (ref: low) } \\
\hline High & $2.719 * \star$ & $\begin{array}{l}(1.739- \\
4.251)\end{array}$ & 2.261 & $\begin{array}{l}(0.673- \\
7.597)\end{array}$ \\
\hline \multicolumn{5}{|c|}{ Perceived severity of COVID-19 (ref: mild) } \\
\hline Severe or moderate & 1.006 & $\begin{array}{l}(0.792- \\
1.278)\end{array}$ & 0.829 & $\begin{array}{l}(0.486- \\
1.417)\end{array}$ \\
\hline \multicolumn{5}{|l|}{${ }^{*} \mathrm{p}<0.05 .{ }^{* *} \mathrm{p}<0.01$} \\
\hline Abbreviation: $\mathrm{aOR}=$ adjust & ds ratio. & & & \\
\hline
\end{tabular}

The uptake of COVID-19 vaccination differed across socio-demographic characteristics, awareness of and perceived susceptibility to COVID-19 (Table 3). Among the 2244 respondents, there were significant differences in the uptake of COVID-19 vaccines by location, age, educational attainment, occupation, annual income, and COVID-19 awareness. When participant's occupations were considered, the percentages of respondents who had not been or scheduled a vaccination ranged from $53.10 \%(411 / 774)$ 
for those working in government agencies to $92.72 \%$ (280/302) for those working in the manufacturing industry or agriculture. Respondents with low perceived susceptibility to COVID-19 were more likely to have not received or scheduled a COVID-19 vaccine $(78.60 \%, 1649 / 2098)$, while those with high perceived susceptibility had a higher uptake rate of COVID-19 vaccinations $(55.84 \%, 81 / 146)$. Regarding willingness to receive COVID-19 vaccinations among the 1711 respondents who had not been vaccinated or did not have vaccinations scheduled, 49.12\% (419/853) from Nanjing city and 54.66\% (469/858) from Chizhou city reported being willing to receive the vaccination. A large proportion of respondents in both the high and low perceived susceptibility to COVID-19 categories were willing to receive a vaccination, $65.63 \%$ (42/64) and 51.37\% (846/1647), respectively. Respondents with high COVID-19 awareness were more willing to receive COVID-19 vaccination $(54.20 \%, 851 / 1570)$, while those with low awareness had a lower willingness to receive COVID-19 vaccination $(26.24 \%, 37 / 141)$. 
Table 3

Characteristics of study respondents by the willingness and uptake of COVID-19 vaccination

\section{Characteristics}

Uptake of COVID-19 vaccination
Willingness of COVID-19 vaccination among those not vaccinated

Willing (\%) Unwilling (\%)
Vaccinated
/scheduled

(\%)

\section{Total}

City

Nanjing

Chizhou

Gender

Male

Female

Age (years)

18-25

26-35

36-45

$>=46$

Residency

Local residents

Migrants

Marital status

Single

Married

Divorced/widow

Educational attainment

Middle school or lower
530 (23.62)

$\mathrm{P}=0.040$

237 (21.72)

293 (25.41)

$\mathrm{P}=0.252$

176 (25.14)

354 (22.93)

$\mathrm{P}<0.001$

46 (21.10)

183 (17.55)

$186(32.80)$

115 (27.64)

$\mathrm{P}=0.499$

$464(23.86)$

66 (22.07)

$\mathrm{P}=0.667$

70 (24.73)

446 (23.34)

$14(28)$

$\mathrm{P}<0.001$

$33(9.68)$
308 (90.32)

Not

vaccinated/scheduled

(\%)

1714 (76.38)

854 (78.28)

860 (74.59)

$524(74.86)$

1190 (77.07)

$172(78.90)$

860 (82.45)

381 (67.20)

301 (72.36)

1481 (76.14)

$233(77.93)$

$213(75.27)$

1465 (76.66)

$36(72)$

$308(90.32)$

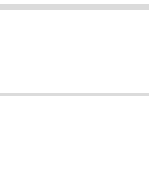

888 (51.90)

$823(48.10)$

$\mathrm{P}=0.022$

$\begin{array}{ll}419(49.12) & 434(50.88) \\ 469(54.66) & 389(45.34)\end{array}$

$\mathrm{P}=0.082$

288 (55.07) 235 (44.93)

600 (50.51) 588 (49.49)

$\mathrm{P}=0.799$

90 (52.33)

$82(47.67)$

454 (52.98) 403 (47.02)

$193(50.66) \quad 188$ (49.34)

151 (50.17) $150(49.83)$

$\mathrm{P}=0.822$

$766(51.79) \quad 713(48.21)$

$122(52.59) \quad 110(47.41)$

$\mathrm{P}=0.277$

$113(53.05) \quad 100(46.95)$

$761(52.05) \quad 701(47.95)$

$14(38.89) \quad 22(61.11)$

$P=0.784$

Note: Only 1714 participants who haven't received or scheduled a COVID-19 vaccine were asked their willingness. Among them, three participants didn't report their willingness, so the sample size of the vaccination willingness question was limited to 1711. 


\section{Characteristics}

Uptake of COVID-19 vaccination

Not

vaccinated/scheduled

(\%)

Vaccinated
/scheduled

(\%)

High school or

technical secondary

$53(13.18)$

349 (86.82)

school

Junior college

149 (26.70)

409 (73.30)

648 (68.72)

$295(31.28)$

higher

Occupation

$P<0.001$

Government agency

Service industry

Manufacturing

industry or agriculture

Others

Annual individual

income

$<20 k$

37 (15.16)

81 (21.72)

173 (24.68)

151 (24.92)

88 (27.50)

$\mathrm{P}=0.072$

544 (92.52)

411 (53.10)

479 (82.59)

280 (92.72)

44 (7.48)

$P=0.007$

20-50k

50-100k

$100-200 k$

$>200 k$

Self-reported health

status

Good

Poor

Awareness of COVID-

19

High

Low

$510(24.50)$
$20(12.35)$

1572 (75.50)

142 (87.65)

207 (84.84)

292 (78.28)

528 (75.32)

455 (75.08)

232 (72.50)

489 (24.15)

1536 (75.85)

178 (81.28)

$P<0.001$

Note: Only 1714 participants who haven't received or scheduled a COVID-19 vaccine were asked their willingness. Among them, three participants didn't report their willingness, so the sample size of the vaccination willingness question was limited to 1711.
$188(54.18)$

$159(45.82)$

$107(52.20) \quad 98(47.80)$

Willingness of COVID-19 vaccination among those not vaccinated

Willing (\%) Unwilling (\%) $209(51.10) \quad 200(48.90)$ $336(51.85) \quad 312(48.15)$

$\mathrm{P}=0.321$

$221(53.77) \quad 190(46.23)$

251 (52.51) 227 (47.49)

$152(54.29) \quad 128(45.71)$

264 (48.71) 278 (51.29)

$\mathrm{P}=0.973$

151 (51.71) 141 (48.29)

$270(51.14) \quad 258(48.86)$

235 (51.76) 219 (48.24)

$125(53.88) \quad 107(46.12)$

$\mathrm{P}=0.050$

$808(52.71) \quad 725(47.29)$

$80(44.94) \quad 98(55.06)$

$P<0.001$

$851(54.20) \quad 719(45.80)$

37 (26.24) 104 (73.76) 


\begin{tabular}{|c|c|c|c|c|}
\hline \multirow[t]{2}{*}{ Characteristics } & \multicolumn{2}{|c|}{ Uptake of COVID-19 vaccination } & \multicolumn{2}{|c|}{$\begin{array}{l}\text { Willingness of COVID-19 } \\
\text { vaccination among those not } \\
\text { vaccinated }\end{array}$} \\
\hline & $\begin{array}{l}\text { Vaccinated } \\
\text { /scheduled } \\
\text { (\%) }\end{array}$ & $\begin{array}{l}\text { Not } \\
\text { vaccinated/scheduled } \\
(\%)\end{array}$ & Willing (\%) & Unwilling (\%) \\
\hline $\begin{array}{l}\text { Perceived } \\
\text { susceptibility of } \\
\text { COVID-19 }\end{array}$ & \multicolumn{2}{|l|}{$P<0.001$} & \multicolumn{2}{|l|}{$P=0.025$} \\
\hline High & $81(55.48)$ & $65(44.52)$ & $42(65.63)$ & $22(34.38)$ \\
\hline Low & $449(21.40)$ & $1649(78.60)$ & $846(51.37)$ & $801(48.63)$ \\
\hline $\begin{array}{l}\text { Perceived severity of } \\
\text { COVID-19 }\end{array}$ & \multicolumn{2}{|l|}{$P=0.103$} & \multicolumn{2}{|l|}{$P=0.163$} \\
\hline Severe or moderate & $113(26.65)$ & $311(73.35)$ & $172(55.48)$ & $138(44.52)$ \\
\hline Mild & $417(22.91)$ & 1403 (77.09) & $716(51.11)$ & $685(48.89)$ \\
\hline \multicolumn{5}{|c|}{$\begin{array}{l}\text { Note: Only } 1714 \text { participants who haven't received or scheduled a COVID-19 vaccine were asked their } \\
\text { willingness. Among them, three participants didn't report their willingness, so the sample size of the } \\
\text { vaccination willingness question was limited to } 1711 \text {. }\end{array}$} \\
\hline
\end{tabular}

Table 4 presents the factors associated with willingness to receive and uptake of COVID-19 vaccinations in the study sites. Location, age, residence, educational attainment, occupation, self-reported health status, and perceived susceptibility to COVID-19 were significantly associated with uptake of COVID-19 vaccination among the survey respondents. Respondents living in Chizhou city, compared to Nanjing city, had a higher uptake rate of COVID-19 vaccination ( $a O R=1.928,95 \% \mathrm{Cl}=1.488-2.498)$. Persons aged 46 years or older ( $\mathrm{aOR}=2.012,95 \% \mathrm{Cl}=1.133-3.574)$, compared to those aged 18-25 years, were more likely to receive or schedule a vaccination, while respondents who had poor perceived health were less likely to receive or schedule a vaccination $(\mathrm{aOR}=0.540,95 \% \mathrm{Cl}=0.352-0.829)$. Vaccination uptake among migrants was 1.479 times $(95 \% \mathrm{Cl}=1.040-2.104)$ higher than among local residents. Respondents who had educational attainment of junior college or higher and worked in government agencies had higher uptake of COVID-19 vaccinations than those with lower educational attainment or less secure occupations (i.e., industry). The vaccine uptake rate among respondents with high perceived susceptibility to COVID-19 was 3.457 times (95\% $\mathrm{Cl}=2.298-5.199)$ higher than those with low perceived susceptibility. In terms of willingness to receive COVID-19 vaccination, among 1711 respondents who had not been or scheduled a vaccination, more respondents in Chizhou city reported being willing to receive a COVID-19 vaccination than those living in Nanjing city $(\mathrm{aOR}=1.404,95 \% \mathrm{Cl}=1.110-1.776)$. Willingness to be vaccinated among respondents with high awareness of and perceived susceptibility to COVID-19 was 3.391 ( $95 \% \mathrm{Cl}=2.285$ 5.032) and 1.950 (95\% $\mathrm{Cl}=1.119-3.398)$ times higher than those with low awareness and perceived susceptibility, respectively. Other socio-demographic characteristics were not associated with the willingness to receive a COVID-19 vaccination among those who had been vaccinated. 
Table 4

Factors associated with the willingness and uptake of COVID-19 vaccination

\begin{tabular}{|c|c|c|c|c|}
\hline & $\begin{array}{l}\text { Uptake of COVID-19 } \\
\text { vaccination (aOR) }\end{array}$ & $95 \% \mathrm{Cl}$ & $\begin{array}{l}\text { Willingness of COVID-19 } \\
\text { vaccination (aOR) }\end{array}$ & $95 \% \mathrm{Cl}$ \\
\hline \multicolumn{5}{|l|}{ City (ref:Nanjing) } \\
\hline Chizhou & $1.928 * \star$ & $\begin{array}{l}(1.488- \\
2.498)\end{array}$ & $1.404 * \star$ & $\begin{array}{l}(1.110- \\
1.776)\end{array}$ \\
\hline \multicolumn{5}{|l|}{ Gender (ref: male) } \\
\hline Female & 0.999 & $\begin{array}{l}(0.781- \\
1.278)\end{array}$ & 0.867 & $\begin{array}{l}(0.694- \\
1.083)\end{array}$ \\
\hline \multicolumn{5}{|l|}{ Age (ref: $18-25$ years) } \\
\hline $26-35$ & 0.713 & $\begin{array}{l}(0.436- \\
1.166)\end{array}$ & 1.024 & $\begin{array}{l}(0.693- \\
1.512)\end{array}$ \\
\hline $36-45$ & 1.680 & $\begin{array}{l}(0.979- \\
2.882)\end{array}$ & 0.953 & $\begin{array}{l}(0.607- \\
1.495)\end{array}$ \\
\hline$>=46$ & $2.012^{\star}$ & $\begin{array}{l}(1.133- \\
3.574)\end{array}$ & 0.946 & $\begin{array}{l}(0.588- \\
1.521)\end{array}$ \\
\hline \multicolumn{5}{|c|}{ Residency (ref: local residents) } \\
\hline Migrants & $1.479 *$ & $\begin{array}{l}(1.040- \\
2.104)\end{array}$ & 1.107 & $\begin{array}{l}(0.818- \\
1.499)\end{array}$ \\
\hline \multicolumn{5}{|l|}{ Marital status (ref: single) } \\
\hline Married & 0.761 & $\begin{array}{l}(0.500- \\
1.159)\end{array}$ & 0.897 & $\begin{array}{l}(0.624- \\
1.290)\end{array}$ \\
\hline Divorced/widow & 1.092 & $\begin{array}{l}(0.479- \\
2.490)\end{array}$ & 0.567 & $\begin{array}{l}(0.262- \\
1.224)\end{array}$ \\
\hline \multicolumn{5}{|c|}{ Educational attainment (ref: middle school or lower) } \\
\hline $\begin{array}{l}\text { High school or technical } \\
\text { secondary school }\end{array}$ & 1.085 & $\begin{array}{l}(0.652- \\
1.807)\end{array}$ & 1.206 & $\begin{array}{l}(0.873- \\
1.667)\end{array}$ \\
\hline Junior college & $1.996^{* *}$ & $\begin{array}{l}(1.236- \\
3.225)\end{array}$ & 1.023 & $\begin{array}{l}(0.734- \\
1.425)\end{array}$ \\
\hline $\begin{array}{l}\text { Bachelor's degree or } \\
\text { higher }\end{array}$ & $2.473^{\star \star}$ & $\begin{array}{l}(1.494- \\
4.094)\end{array}$ & 1.024 & $\begin{array}{l}(0.716- \\
1.465)\end{array}$ \\
\hline
\end{tabular}

${ }^{*} p<0.05 .{ }^{* *} p<0.01$.

Abbreviation: aOR=adjusted odds ratio. 


\begin{tabular}{|c|c|c|c|c|}
\hline & $\begin{array}{l}\text { Uptake of COVID-19 } \\
\text { vaccination (aOR) }\end{array}$ & $95 \% \mathrm{Cl}$ & $\begin{array}{l}\text { Willingness of COVID-19 } \\
\text { vaccination (aOR) }\end{array}$ & $95 \% \mathrm{Cl}$ \\
\hline Service industry & $0.285^{\star \star}$ & $\begin{array}{l}(0.214- \\
0.381)\end{array}$ & 1.010 & $\begin{array}{l}(0.756- \\
1.348)\end{array}$ \\
\hline $\begin{array}{l}\text { Manufacturing industry } \\
\text { or agriculture }\end{array}$ & $0.104 \star \star$ & $\begin{array}{l}(0.0630 \\
-0.171)\end{array}$ & 1.008 & $\begin{array}{l}(0.718- \\
1.416)\end{array}$ \\
\hline Others & $0.119 * \star$ & $\begin{array}{r}(0.0809 \\
-0.175)\end{array}$ & 0.846 & $\begin{array}{l}(0.626- \\
1.145)\end{array}$ \\
\hline \multicolumn{5}{|c|}{ Annual individual income (ref: <20k) } \\
\hline $20-50 k$ & 1.487 & $\begin{array}{l}(0.909- \\
2.432)\end{array}$ & 0.939 & $\begin{array}{l}(0.650- \\
1.358)\end{array}$ \\
\hline $50-100 k$ & 1.061 & $\begin{array}{l}(0.672- \\
1.677)\end{array}$ & 0.905 & $\begin{array}{l}(0.644- \\
1.273)\end{array}$ \\
\hline $100-200 k$ & 0.719 & $\begin{array}{l}(0.445- \\
1.163)\end{array}$ & 0.955 & $\begin{array}{l}(0.661- \\
1.379)\end{array}$ \\
\hline$>200 \mathrm{k}$ & 0.747 & $\begin{array}{l}(0.441- \\
1.265)\end{array}$ & 1.106 & $\begin{array}{l}(0.724- \\
1.689)\end{array}$ \\
\hline \multicolumn{5}{|c|}{ Self-reported health status (ref: good) } \\
\hline Poor & $0.540 \star \star$ & $\begin{array}{l}(0.352- \\
0.829)\end{array}$ & 0.757 & $\begin{array}{l}(0.543- \\
1.056)\end{array}$ \\
\hline \multicolumn{5}{|c|}{ Awareness of COVID-19 (ref: low) } \\
\hline High & 1.683 & $\begin{array}{l}(0.991- \\
2.859)\end{array}$ & $3.391^{\star \star}$ & $\begin{array}{l}(2.285- \\
5.032)\end{array}$ \\
\hline \multicolumn{5}{|c|}{ Perceived susceptibility of COVID-19 (ref: low) } \\
\hline High & $3.457^{\star \star}$ & $\begin{array}{l}(2.298- \\
5.199)\end{array}$ & $1.950^{\star}$ & $\begin{array}{l}(1.119- \\
3.398)\end{array}$ \\
\hline \multicolumn{5}{|c|}{ Perceived severity of COVID-19 (ref: mild) } \\
\hline Severe or moderate & 0.844 & $\begin{array}{l}(0.629- \\
1.133)\end{array}$ & 1.213 & $\begin{array}{l}(0.933- \\
1.578)\end{array}$ \\
\hline \multicolumn{5}{|l|}{${ }^{\star} p<0.05 .{ }^{* *} p<0.01$} \\
\hline Abbreviation: $\mathrm{aOR}=$ adjus & odds ratio. & & & \\
\hline
\end{tabular}

\section{Discussion}

By early 2021, 52.45\% of adults aged 18 years and older had received or scheduled a COVID-19 test at least once, and $95.63 \%$ of total respondents reported being willing to receive a test in the future; $63.28 \%$ of respondents had received/scheduled a vaccination or were willing to be vaccinated against COVID-19 
in the future. Higher willingness and uptake of COVID-19 testing were associated with more secure occupations, while associations with other socio-demographic characteristics failed to reach statistical significance. Being of older age and migrants, having higher educational attainment and working in a secure job were associated with higher uptake of COVID-19 vaccinations, while willingness to receive a vaccination was consistent across the various socio-demographic characteristics assessed. High awareness of and perceived susceptibility to COVID-19 were associated with a higher willingness to receive and uptake COVID-19 testing and vaccination.

Adults in China expressed almost universal willingness to receive COVID-19 testing (95.63\%); this rate is higher than populations observed in some other countries, such as Ethiopian and Japan. ${ }^{58,59}$ Regarding the uptake of COVID-19 testing, although some areas had not reported any confirmed cases, over half of respondents reported having received or scheduled a test. Except for occupation, no socio-demographic characteristic was associated with the willingness and uptake of COVID-19 testing, which is consistent with previous studies. ${ }^{51,60}$ This indicated the equal willingness and access to COVID-19 testing in China, which may lie in that the Chinese central government and some local governments require healthcare providers to supply the community-wide mass COVID-19 testing without cost-sharing and the testing policies for travel. ${ }^{61}$ As a result, we found that the majority of tests were performed due to communitywide mass testing and mandatory testing policies for travel. This finding provides some evidence to support the rollout of mass-testing in urban regions of China. The different uptake rate by occupation may result from the requirements of the employers and government, which may alleviate the anxiety to go back to work in person and be assist in preventing and controlling confirmed cases as well. During the current phase of the pandemic with only sporadic cases, mass testing policies appear to be supported by the public, and continuous implementing these policies could help identify, trace and mitigate confirmed cases even during the low-risk period of the COVID-19 pandemic in China.

As China entered the low-risk period of the COVID-19 pandemic, willingness of the COVID-19 vaccination appears to become relatively low, with only $63.28 \%$ of total respondents having received/scheduled or were willing to receive a vaccination, which could pose a challenge to the achievement of herd immunity. The willingness of COVID-19 vaccination in our study is lower than that in mid-2020 in China. ${ }^{46}$ This reduced willingness to vaccinate could be due to the successful prevention and control of the pandemic and low risks of COVID-19 infections in China. ${ }^{4}$ Concern regarding the efficacy and safety of the COVID19 vaccine may be another reason for individuals not being willing to receive a vaccination. ${ }^{62}$ Thus, the importance, efficacy, and safety of COVID-19 vaccines should be emphasized more to maintain high willingness of vaccination especially when the pandemic evolved into the low-risk period. In fact, vaccination willingness had been observed to rebound with outbreaks in several cities in $2021^{63}$. For example, following the outbreak in Guangzhou city in May 2021, uptake of both doses of the COVID-19 vaccination has quickly reached $70 \%$ within a month in Guangzhou ${ }^{64}$ and nearby Shenzhen ${ }^{65}$.

Among adults who had not been vaccinated or scheduled a vaccination, socio-demographic characteristics were not associated with willingness to receive a vaccination, indicating the equitable 
vaccination willingness in China. ${ }^{21,40}$ However, the uptake of vaccinations differed by age, residence, educational attainment, and occupation. These disparities are aligned with previous studies in other countries that reported a higher vaccination uptake among older adults and those with higher

socioeconomic status. ${ }^{28,29,31,36}$ In China, the COVID-19 vaccination is free to the public, ${ }^{66}$ and to improve the access to the vaccination, COVID-19 vaccination units are temporarily set up within the community providing walk-up COVID-19 vaccination ${ }^{67}$. Older adults and migrants face more risk of infecting COVID19 , and are the priority groups of vaccination. Although with equitable willingness, population with a higher level of educational attainment and working in a secure job faced more policy requirements and less barriers to be vaccinated than their counterparts. Most of them work at government agency and formal employment, which usually require the employees to be fully vaccinated before returning to the office in person. This policy requirements may contribute to the higher uptake of vaccination among those with a higher level of educational attainment and working in a secure job.

In addition, higher awareness of and perceived susceptibility to COVID-19 were positively associated with the respondent's willingness to receive and uptake the COVID-19 test and vaccination, concurs with data from previous studies that report perceiving a high risk of infections may increase willingness and uptake rates for both testing and vaccination. ${ }^{29,33,42-47,51,60,68,69}$

This study is subject to several limitations. First, this study conducted an online survey, which may have resulted in the study groups being more homogenous with respect to certain socio-demographics. Second, this cross-sectional study just showed associations, instead of causal associations, and did not compare the current rates of willingness to receive and uptake of COVID-19 testing and vaccination with the rates from the active period of the pandemic, and therefore cannot capture trends or the changes in these proportions over time. Last, this study was conducted at the early stage of COVID-19 vaccination rollout. Thus, further research is warranted to assess the public's uptake and willingness to receives COVID-19 test and vaccines in different phases of the pandemic.

\section{Conclusion}

Chinese adults expressed almost universal willingness of COVID-19 testing and relatively low willingness of COVID-19 vaccination at the low-risk period of the COVID-19 pandemic. The willingness and uptake of COVID-19 testing and vaccination are independent from their socio-demographic characteristics at most cases.

\section{Declarations}

\section{Contribution}

ZH designed this study. LG and CX collected the data. SZ conducted literature review. SS analyzed the data and wrote the manuscript. ZH, LL, and MF critically reviewed and revised the manuscript. All authors have seen and approved the final version of the manuscript. 


\section{Funding}

ZH acknowledges financial support from the National Natural Science Foundation of China (No. 71874034) and the Three-Year Initiative Plan for Strengthening Public Health System Construction in Shanghai (2020-2022) (GWV-10.1-XK23). The authors thank Zhiqiang Qu from the School of Public Health, Fudan University for his help in cleaning data. The funders had no role in study design, data collection and analysis, decision to publish, or preparation of the manuscript.

\section{Declaration of Competing Interest}

None.

\section{References}

1. M. M. Alvi, S. Sivasankaran, M. Singh. Pharmacological and non-pharmacological efforts at prevention, mitigation, and treatment for COVID-19. Journal of Drug Targeting. 2020;28(7-8):742754. http://doi.org/10.1080/1061186X.2020.1793990

2. S. Dryden-Peterson, G. E. Velásquez, T. J. Stopka, et al. Disparities in SARS-CoV-2 Testing in Massachusetts During the COVID-19 Pandemic. JAMA network open. 2021;4(2):e2037067. http://doi.org/10.1001/jamanetworkopen.2020.37067

3. M. J. Pletcher, J. E. Olgin, N. D. Peyser, et al. Factors Associated With Access to and Timing of Coronavirus Testing Among US Adults After Onset of Febrile Illness. JAMA network open. 2021;4(5):e218500. http://doi.org/10.1001/jamanetworkopen.2021.8500

4. J. Wang, X. Lu, X. Lai, et al. The Changing Acceptance of COVID-19 Vaccination in Different Epidemic Phases in China: A Longitudinal Study. Vaccines (Basel). 2021;9(3).

http://doi.org/10.3390/vaccines9030191

5. E. Gambarini, M. Galli, D. Di Nardo, et al. A Survey on Perceived COVID-19 Risk in Dentistry and the Possible Use of Rapid Tests. J Contemp Dent Pract. 2020;21(7):718-722.

6. L. S. F. Frederiksen, Y. Zhang, C. Foged, A. Thakur. The Long Road Toward COVID-19 Herd Immunity: Vaccine Platform Technologies and Mass Immunization Strategies. Front Immunol. 2020;11:18171817. http://doi.org/10.3389/fimmu.2020.01817

7. CCTV. Dialogue. 2020; https://tv.cctv.com/2020/08/22/VIDE7gljYRxsD6J7KZuF6yKW200822.shtml? spm=C22284.P87019257382.EMqe9pBD7J5t.150. Accessed 13 August, 2021.

8. T. S. C. I. O. o. t. P. s. R. o. China. Joint prevention and control mechanism of the state council: Conditional listing of new Coronavirus vaccine. 2020; http://www.scio.gov.cn/video/42600/42601/Document/1696292/1696292.htm. Accessed 15 August, 2021.

9. G. Zhou. COVID-19 vaccine authorities. Chinese Journalist. 2021(03):120-124.

10. Press Conference of the Joint Prevention and Control Mechanism of the State Council on March 12, 2020. http://www.gov.cn/xinwen/gwylflkjz54/index.htm. Accessed 9 August, 2021. 
11. China: WHO Coronavirus Disease (COVID-19) Dashboard. https://covid19.who.int/region/wpro/country/cn/. Accessed 9 August, 2021.

12. G. Bai. Timeline of new outbreak in Xinfadi. Beijing. Beijing Daily. 2020;6(19).

13. C. Qi; J. Zhen. Investigation on the source of the epidemic in Qingdao. Beijing News. 2020;10(16).

14. R. Al-Amer, D. Maneze, B. Everett, et al. COVID-19 vaccination intention in the first year of the pandemic: A systematic review. J Clin Nurs. 2021. http://doi.org/10.1111/jocn.15951

15. G. F. Al-Jayyousi, M. A. M. Sherbash, L. A. M. Ali, et al. Factors Influencing Public Attitudes towards COVID-19 Vaccination: A Scoping Review Informed by the Socio-Ecological Model. Vaccines (Basel). 2021;9(6). http://doi.org/10.3390/vaccines 9060548

16. L. K. Kociolek, J. Elhadary, R. Jhaveri, et al. Coronavirus disease 2019 vaccine hesitancy among children's hospital staff: A single-center survey. Infect Control Hosp Epidemiol. 2021;42(6):775-777. http://doi.org/10.1017/ice.2021.58

17. D. Almaghaslah, A. Alsayari, G. Kandasamy, R. Vasudevan. COVID-19 Vaccine Hesitancy among Young Adults in Saudi Arabia: A Cross-Sectional Web-Based Study. Vaccines (Basel). 2021;9(4). http://doi.org/10.3390/vaccines9040330

18. R. M. Ghazy, S. Abd ElHafeez, R. Shaaban, et al. Determining the Cutoff Points of the $5 \mathrm{C}$ Scale for Assessment of COVID-19 Vaccines Psychological Antecedents among the Arab Population: A Multinational Study. J Prim Care Community Health. 2021;12:21501327211018568. http://doi.org/10.1177/21501327211018568

19. F. Guaraldi, M. Montalti, Z. Di Valerio, et al. Rate and Predictors of Hesitancy toward SARS-CoV-2 Vaccine among Type 2 Diabetic Patients: Results from an Italian Survey. Vaccines (Basel). 2021;9(5). http://doi.org/10.3390/vaccines 9050460

20. A. Kecojevic, C. H. Basch, M. Sullivan, Y. T. Chen, N. K. Davi. COVID-19 Vaccination and Intention to Vaccinate Among a Sample of College Students in New Jersey. J Community Health. 2021:1-10. http://doi.org/10.1007/s10900-021-00992-3

21. R. Kuhn, B. Henwood, A. Lawton, et al. COVID-19 vaccine access and attitudes among people experiencing homelessness from pilot mobile phone survey in Los Angeles, CA. PLOS One. 2021;16(7):e0255246. http://doi.org/10.1371/journal.pone.0255246

22. D. T. Smith, K. Attwell, U. Evers. Support for a COVID-19 vaccine mandate in the face of safety concerns and political affiliations: An Australian study. Politics. 2021:02633957211009066. http://doi.org/10.1177/02633957211009066

23. P. Vishani, P. Krishani. Study of Awareness Regarding Covid-19 Vaccines Among General Population. Research Square. 2021. http://doi.org/10.21203/rs.3.rs-537278/v1

24. K. Andrejko, J. Pry, J. F. Myers, et al. Early evidence of COVID-19 vaccine effectiveness within the general population of California. medRxiv. 2021:2021.2004.2008.21255135. http://doi.org/10.1101/2021.04.08.21255135

25. M. Barry, M.-H. Temsah, F. Aljamaan, et al. COVID-19 vaccine uptake among healthcare workers in the fourth country to authorize BNT162b2 during the first month of rollout. medRxiv. 
2021:2021.2001.2029.21250749. http://doi.org/10.1101/2021.01.29.21250749

26. Z. Hou, S. Song, F. Du, et al. The Influence of the COVID-19 Epidemic on Prevention and Vaccination Behaviors Among Chinese Children and Adolescents: Cross-sectional Online Survey Study. JMIR Public Health Surveill. 2021;7(5):e26372. http://doi.org/10.2196/26372

27. Y. A. Adebisi, A. J. Alaran, O. A. Bolarinwa, W. Akande-Sholabi, D. E. Lucero-Prisno. When it is available, will we take it? Social media users' perception of hypothetical COVID-19 vaccine in Nigeria. Pan Afr Med J. 2021;38:230. http://doi.org/10.11604/pamj.2021.38.230.27325

28. B. N. Baack, N. Abad, D. Yankey, et al. COVID-19 Vaccination Coverage and Intent Among Adults Aged 18-39 Years - United States, March-May 2021. MMWR Morb Mortal Wkly Rep. 2021;70(25):928-933. http://doi.org/10.15585/mmwr.mm7025e2

29. A. B. Coe, M. H. Elliott, S. B. S. Gatewood, J. R. Goode, L. R. Moczygemba. Perceptions and predictors of intention to receive the COVID-19 vaccine. Res Social Adm Pharm. 2021. http://doi.org/10.1016/j.sapharm.2021.04.023

30. Y. Lin, Z. Hu, Q. Zhao, et al. Understanding COVID-19 vaccine demand and hesitancy: A nationwide online survey in China. PLoS Negl Trop Dis. 2020;14(12):e0008961. http://doi.org/10.1371/journal.pntd.0008961

31. A. A. Malik, S. M. McFadden, J. Elharake, S. B. Omer. Determinants of COVID-19 vaccine acceptance in the US. EClinicalMedicine. 2020;26:100495. http://doi.org/10.1016/j.eclinm.2020.100495

32. L. H. Nguyen, A. D. Joshi, D. A. Drew, et al. Racial and ethnic differences in COVID-19 vaccine hesitancy and uptake. medRxiv. 2021. http://doi.org/10.1101/2021.02.25.21252402

33. L. Tao, R. Wang, N. Han, et al. Acceptance of a COVID-19 vaccine and associated factors among pregnant women in China: a multi-center cross-sectional study based on health belief model. Hum Vaccin Immunother. 2021:1-10. http://doi.org/10.1080/21645515.2021.1892432

34. L. Tao, R. Wang, J. Liu. Comparison of Vaccine Acceptance Between COVID-19 and Seasonal Influenza Among Women in China: A National Online Survey Based on Health Belief Model. Front Med (Lausanne). 2021;8:679520. http://doi.org/10.3389/fmed.2021.679520

35. B. Akarsu, D. Canbay Özdemir, D. Ayhan Baser, et al. While studies on COVID-19 vaccine is ongoing, the public's thoughts and attitudes to the future COVID-19 vaccine. Int J Clin Pract. 2021;75(4):e13891. http://doi.org/10.1111/ijcp.13891

36. M. M. Akiful Haque, M. L. Rahman, M. Hossian, et al. Acceptance of COVID-19 vaccine and its determinants: evidence from a large sample study in Bangladesh. Heliyon. 2021;7(6):e07376. http://doi.org/10.1016/j.heliyon.2021.e07376

37. S. Al-Marshoudi, H. Al-Balushi, A. Al-Wahaibi, et al. Knowledge, Attitudes, and Practices (KAP) toward the COVID-19 Vaccine in Oman: A Pre-Campaign Cross-Sectional Study. Vaccines (Basel). 2021;9(6). http://doi.org/10.3390/vaccines9060602

38. A. H. Al-Mistarehi, K. A. Kheirallah, A. Yassin, et al. Determinants of the willingness of the general population to get vaccinated against COVID-19 in a developing country. Clin Exp Vaccine Res. 2021;10(2):171-182. http://doi.org/10.7774/cevr.2021.10.2.171 
39. A. A. Dror, N. Eisenbach, S. Taiber, et al. Vaccine hesitancy: the next challenge in the fight against COVID-19. Eur J Epidemiol. 2020;35(8):775-779. http://doi.org/10.1007/s10654-020-00671-y

40. K. Han, M. R. Francis, R. Zhang, et al. Confidence, Acceptance and Willingness to Pay for the COVID19 Vaccine among Migrants in Shanghai, China: A Cross-Sectional Study. Vaccines (Basel). 2021;9(5). http://doi.org/10.3390/vaccines9050443

41. C. La Vecchia, E. Negri, G. Alicandro, V. Scarpino. Attitudes towards influenza vaccine and a potential COVID-19 vaccine in Italy and differences across occupational groups, September 2020. Med Lav. 2020;111(6):445-448. http://doi.org/10.23749/mdl.v111i6.10813

42. R. Abu-Farha, T. Mukattash, R. Itani, et al. Willingness of Middle Eastern Public to Receive COVID-19 Vaccines. Saudi Pharm J. 2021. http://doi.org/10.1016/j.jsps.2021.05.005

43. M. Al-Mohaithef, B. K. Padhi. Determinants of COVID-19 Vaccine Acceptance in Saudi Arabia: A WebBased National Survey. J Multidiscip Healthc. 2020;13:1657-1663. http://doi.org/10.2147/jmdh.S276771

44. M. Detoc, S. Bruel, P. Frappe, et al. Intention to participate in a COVID-19 vaccine clinical trial and to get vaccinated against COVID-19 in France during the pandemic. Vaccine. 2020;38(45):7002-7006. http://doi.org/10.1016/j.vaccine.2020.09.041

45. J. P. D. Guidry, L. I. Laestadius, E. K. Vraga, et al. Willingness to get the COVID-19 vaccine with and without emergency use authorization. Am J Infect Control. 2021;49(2):137-142. http://doi.org/10.1016/j.ajic.2020.11.018

46. J. Wang, R. Jing, X. Lai, et al. Acceptance of COVID-19 Vaccination during the COVID-19 Pandemic in China. Vaccines (Basel). 2020;8(3). http://doi.org/10.3390/vaccines8030482

47. L. P. Wong, H. Alias, P. F. Wong, H. Y. Lee, S. AbuBakar. The use of the health belief model to assess predictors of intent to receive the COVID-19 vaccine and willingness to pay. Hum Vaccin Immunother. 2020;16(9):2204-2214. http://doi.org/10.1080/21645515.2020.1790279

48. S. Y. Lin L, Wang Q, Pu J, Sun FY, Zhang Y, Zhou X, Larson HJ, Hou Z. Public Attitudes and Factors of COVID-19 Testing Hesitancy in the United Kingdom and China: Comparative Infodemiology Study. JMIR Infodemiology 2021;1(1):e26895. http://doi.org/10.2196/26895

49. W. D. S. Almeida, C. L. Szwarcwald, D. C. Malta, et al. Changes in Brazilians' socioeconomic and health conditions during the COVID-19 pandemic. Rev Bras Epidemiol. 2021;23:e200105. http://doi.org/10.1590/1980-549720200105

50. K. C. Fitzgerald, C. A. Mecoli, M. Douglas, et al. Risk factors for infection and health impacts of the COVID-19 pandemic in people with autoimmune diseases. medRxiv. 2021. http://doi.org/10.1101/2021.02.03.21251069

51. T. Vandrevala, A. Montague, P. Terry, M. Fielder. Willingness of the UK public to volunteer for testing in relation to the COVID-19 pandemic. Research Square; 2020.

52. S. Li, B. Feng, W. Liao, W. Pan. Internet Use, Risk Awareness, and Demographic Characteristics Associated With Engagement in Preventive Behaviors and Testing: Cross-Sectional Survey on COVID19 in the United States. J Med Internet Res. 2020;22(6):e19782. http://doi.org/10.2196/19782 
53. T. Quach, L. N. Đoàn, J. Liou, N. A. Ponce. A Rapid Assessment of the Impact of COVID-19 on Asian Americans: Cross-sectional Survey Study. JMIR Public Health Surveill. 2021;7(6):e23976. http://doi.org/10.2196/23976

54. J. A. Tamargo, H. R. Martin, J. Diaz-Martinez, et al. COVID-19 Testing and the Impact of the Pandemic on the Miami Adult Studies on HIV Cohort. J Acquir Immune Defic Syndr. 2021;87(4):1016-1023. http://doi.org/10.1097/qai.0000000000002680

55. J. Hasell, E. Mathieu, D. Beltekian, et al. A cross-country database of COVID-19 testing. Sci Data, 7, 345 (2020). https://ourworldindata.org/coronavirus-testing. Accessed 8 August, 2021.

56. C. C. S. Y. 2020. Urban Socio-Economic Investigation Department, National Bureau of Statistics. Beijing: China Statistics Press; 2021.

57. I. M. Fund. World Economic Outlook (April 2021). 2021; https://www.imf.org/external/datamapper/datasets/WEO. Accessed 15 August, 2021.

58. Y. Belsti, Y. Y. Gela, Y. Akalu, et al. Willingness of Ethiopian Population to Receive COVID-19 Vaccine. J Multidiscip Healthc. 2021;14:1233-1243. http://doi.org/10.2147/jmdh.s312637

59. T. Yoda, H. Katsuyama. Willingness to Receive COVID-19 Vaccination in Japan. Vaccines (Basel). 2021;9(1). http://doi.org/10.3390/vaccines9010048

60. F. Fallucchi, L. Görges, J. Machado, A. Pieters, M. Suhrcke. How to make universal, voluntary testing for COVID-19 work? A behavioural economics perspective. Health Policy. 2021;125(8):972-980. http://doi.org/10.1016/j.healthpol.2021.05.003

61. H. News. The cost of COVID-19 testing in China: the COVID-19 testing is free to these people in some provinces such as Heilong Jiang and Tibet. 2021; https://news.sina.com.cn/c/2021-02-14/docikftpnny6780526.shtml. Accessed August 15, 2021.

62. D. Zhang, Q. Cui, J. Xu, et al. Factors related with COVID-19 vaccination willingness among outpatients in China. Hum Vaccin Immunother. 2021:1-6. http://doi.org/10.1080/21645515.2021.1954441

63. N. H. C. o. t. P. s. R. o. China. Prevention and control of COVID-19. 2021; http://www.nhc.gov.cn/xcs/xxgzbd/gzbd_index.shtml. Accessed 15 August, 2021.

64. P. s. D. Online. Guangzhou vaccine coverage rate has reached $66.6 \%$. 2021; https://baijiahao.baidu.com/s?id=1707313453968214569\&wfr=spider\&for=pc. Accessed 11 August, 2021.

65. B. News. The cumulative number of people vaccinated against the new crown in Shenzhen reached 13.01 million, and the second shot vaccination rate reached $68.9 \%$. 2021; https://baijiahao.baidu.com/s?id=1703261254398754560\&wfr=spider\&for=pc. Accessed 11 August, 2021.

66. C. G. Website. COVID-19 vaccines are free to the public. 2021; http://www.gov.cn/fuwu/202101/09/content_5578430.htm. Accessed August 15, 2021.

67. C. G. Website. Information on the COVID-19 vaccination. 2021; http://www.gov.cn/xinwen/gwylflkjz151/index.htm. Accessed August 15, 2021. 
68. R. Jaspal, G. M. Breakwell. Social support, perceived risk and the likelihood of COVID-19 testing and vaccination: cross-sectional data from the United Kingdom. Curr Psychol. 2021:1-13. http://doi.org/10.1007/s12144-021-01681-z

69. R. D. Ravert, L. Y. Fu, G. D. Zimet. Young Adults' COVID-19 Testing Intentions: The Role of Health Beliefs and Anticipated Regret. J Adolesc Health. 2021;68(3):460-463. http://doi.org/10.1016/j.jadohealth.2020.12.001

\section{Figures}


A
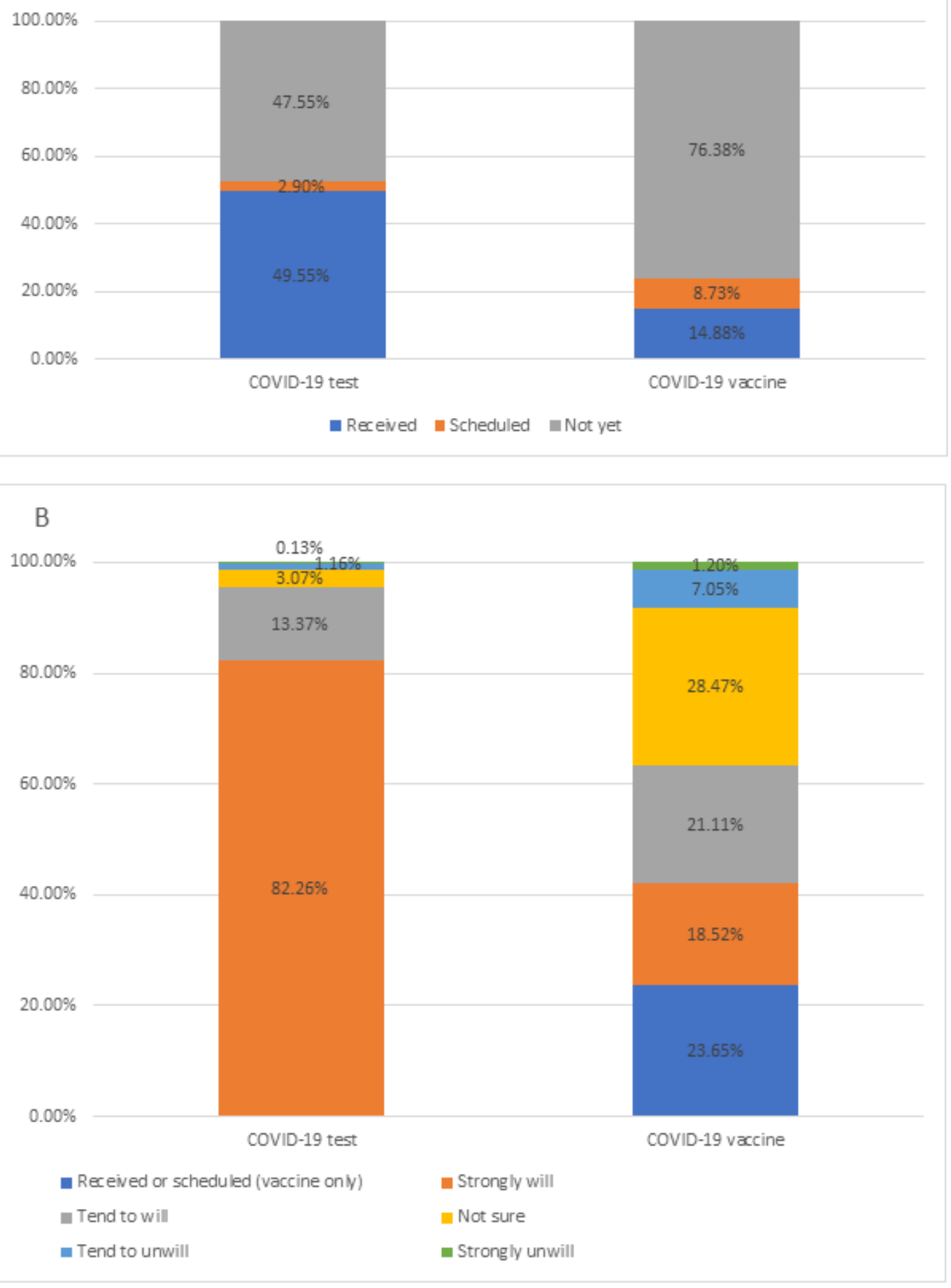

\section{Figure 1}

Willingness and uptake of COVID-19 test and vaccination. Figure 1A. Uptake of COVID-19 test and vaccination. Figure 1B. Willingness of COVID-19 test and vaccination. Note: Willingness of COVID-19 vaccination included 3 missing values. 


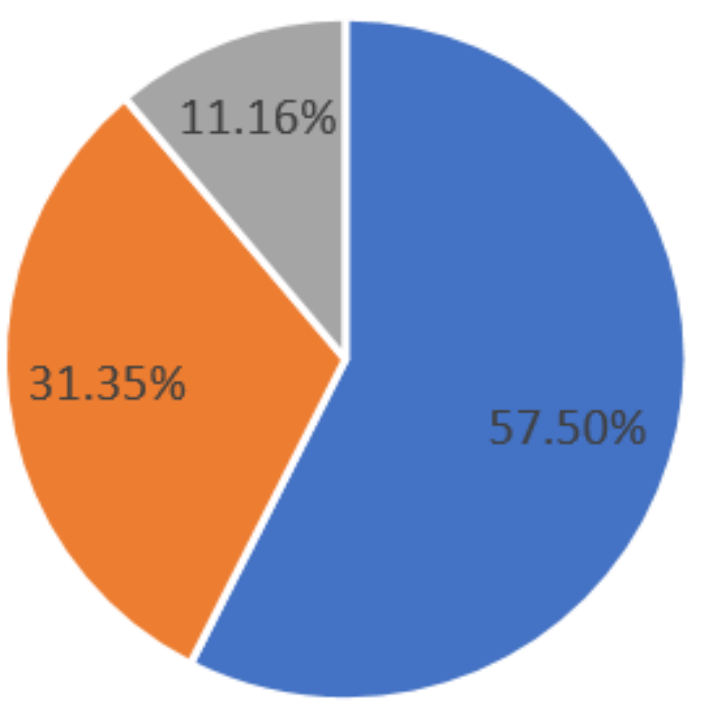

- Community-wide mass testing led by governments

- Mandatory testing policies for travel

- Personal health needs

\section{Figure 2}

Reasons of the uptake of COVID-19 test. Note: Three participants who have received the COVID-19 test didn't report their reasons of receiving the test, so the sample size of the test reason question was limited to 1174 . 